\title{
CONTROL FOR HIGH-SPEED ARCHIMEDEAN SPIRAL NANOPOSITIONING
}

\author{
Andreas Kotsopoulos ${ }^{1}$, Angeliki Pantazi ${ }^{2}$ and Theodore Antonakopoulos ${ }^{1}$ \\ ${ }^{1}$ Department of Electrical and Computers Engineering, \\ University of Patras, 26500, Rio-Patras, Greece \\ E-mail: akotsop, antonako@upatras.gr \\ ${ }^{2}$ IBM Research - Zurich \\ Rüschlikon, Switzerland, CH-8803 \\ E-mail: agp@ zurich.ibm.com
}

\begin{abstract}
Scanning probes are being considered as the basis for a variety of emerging nanoscale applications including sample imaging and ultra-high-density probe storage. In this work, a controller for track-follow in archimedean spiral nanopositioning is presented. The proposed tracking controller is based on a Linear Quadratic Gaussian (LQG) component and a tracking controller, extended with frequency-sliding peak filters in order to exploit the inherent properties of the spiral reference signal. Simulations show that the proposed architecture enables spiral scan operation with very low tracking error even at very high scan frequencies.
\end{abstract}

Index Terms - MEMS, peak, spiral, nanopositioning, probe, control.

\section{INTRODUCTION}

High-speed and accurate positioning is of major significance for rapidly emerging applications that use scanning probe techniques in order to observe, image, manipulate and alter materials down at the nanometer scale. In such systems, nanopositioning is performed using MEMS-based microscanners or piezoscanners [1] with 2-D motion capabilities typically in the order of $100 \mu \mathrm{m}$, which position the medium/sample laterally and longitudinally, relative to probes equipped with nanometer-sharp tips.

Furthermore, the joint requirements of high-speed and nanometer-precise positioning imposed by scanning-probe applications, represent a major challenge for prospective control and nanopositioning schemes. In the majority of today's scanning-probe systems [2],[3], the nanopositioner follows a raster scan or similar trajectory. The implementation of such a motion pattern can be achieved by driving the scanner with a triangular-shaped waveform in the fast axis and a staircase or ramp waveform in the slow axis. Nevertheless, the existence of abrupt changes in direction of motion, produces undesired oscillations and widens the bandwidth of the refer- ence signal. Moreover, operation interruption periods during backward motion towards the start of the next line to scan causes significant drop in the mean data rate.

Recently, an alternative nanopositioning scheme was proposed, based on moving along archimedean spiral trajectories with constant linear velocity, hence maintaining the same positioning resolution throughout the entire trajectory and achieving perpetual system operation [4]. Similar scanning schemes have been used in the Compact Disc (CD) and Digital Versatile Disc (DVD) data storage cases, but with different motion mechanics and positioning accuracy constraints [5].

In order to achieve combined high speed and high precision operation, a new controller for track-follow in spiral nanopositioning has to be developed. The proposed controller is based on the Linear Quadratic Gaussian (LQG) architecture for raster scan positioning used in [6],[7], which is tuned and extended with frequency-sliding peak filters in order to explicitly exploit the variable scan frequency and narrowband frequency spectrum properties of the spiral reference signal. The new architecture has to enable spiral scan operation with very low tracking error even at very high scan frequencies near the end of the controller bandwidth. Note, that higher scan frequencies result to higher probe-storage data-rates or faster imaging operations, for a given controller bandwidth.

The rest of this paper is organized as follows: Section 2 analyzes the spire-wise change in the scan frequency and the key properties of the MEMS microscanner system. In Section 3 the proposed controller architecture is presented, while Section 4 shows performance results, which demonstrate the advantage of the proposed architecture.

\section{SPIRAL NANOPOSITIONING AND MEMS MICROSCANNER MODEL}

In archimedean spiral nanopositioning, motion starts from an initial point of radius $r_{\text {min }}$ from the medium center and follows a spiral trajectory of equidistant turnings around the center with respect to the medium $\mathrm{x} / \mathrm{y}$-plane, until a maximum ra- 


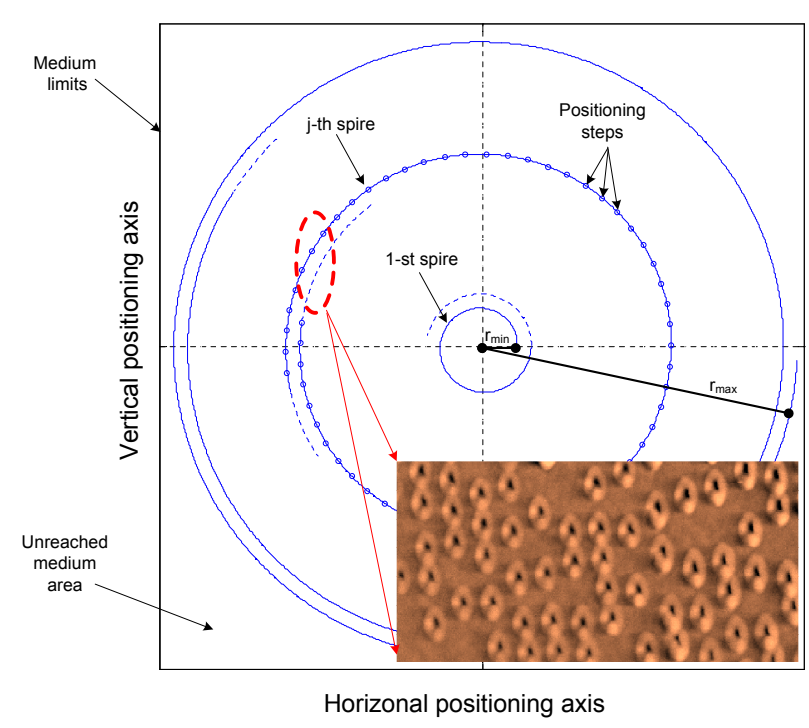

Fig. 1. Overview of spiral nanopositioning and experimental image of spiral engraved indentations on polymer [4].

dius $r_{\max }$ is reached, which is defined by either the medium boundaries or the scanner travel range, as shown in Figure 1. Unlike raster positioning, spiral positioning results in a variable scan frequency when moving along the trajectory with constant linear velocity. In fact, the center scan frequency and bandwidth of the reference positioning signal decreases as motion advances to larger radius values. Note, that an in depth mathematical analysis of constant linear velocity spiral nanopositioning and its properties can be found in [4].

\subsection{Calculation of spire-wise scan frequency}

The length of a plane curve $c=(x, y):(a, b) \rightarrow R^{2}$ is given by $\int_{a}^{b}\left\|c^{\prime}(u)\right\| d u$, where $\|c\|$ denotes the euclidean norm of $c$ with $\|c\|=\sqrt{x^{2}+y^{2}}$ in $R^{2}$. Hence, the length $s$ of the archimedean spiral over the region $(0, \theta)$ can be calculated in closed form as:

$$
s(\theta)=\frac{1}{2} a\left[\theta \sqrt{1+\theta^{2}}+\ln \left(\theta+\sqrt{1+\theta^{2}}\right)\right]
$$

where $\theta$ the polar angle and $2 \pi a$ the interspire distance.

Then, the center spiral scan frequency when moving along the trajectory towards larger radii with constant linear velocity $v$, can be expressed in terms of $v$ and the spire number $j=$ $1,2, \ldots$ as:

$$
f_{c}(j)=\frac{1}{t_{j}}=\frac{1}{v}\left(s\left(\theta_{j}\right)-s\left(\theta_{j-1}\right)\right)
$$

where $\theta_{j-1}=2(j-1) \pi$ and $\theta_{j}=2 j \pi$. It should be noted, that for the above analysis it has been assumed that $r_{\min }=0$ (parameter $b$ of the spiral equations [4]) for simplicity. However, a closed form of $s$ can be readily obtained using the same steps for the generalized case with $b$ in the final expression.

\subsection{MEMS microscanner model}

For the controller design, the reduced second order model of the higher order measured system of the MEMS microscanner [8] analyzed in [6] was used as the plant, while for the performance results, the full system with higher order resonances was used. The magnitude and phase response of the higher order system is depicted in Figure 2, where $P_{x x}, P_{y y}$ denote the system's transfer functions in each individual axes $\mathrm{x}$ and $\mathrm{y}$ respectively. It is clear that the system's dynamic response is dominated by the second order resonances found at approximately $178 \mathrm{~Hz}$ and $158 \mathrm{~Hz}$ for $P_{x x}$ and $P_{y y}$ respectively. Furthermore, the $\mathrm{x} / \mathrm{y}$ position measurement noise is modeled, using the thermal-sensor model described in [6], along with the quantization noise generated at the two 18-bit analog-todigital converters. The standard deviation of thermal-sensor noise was set to $0.8 \mathrm{~nm}$. Finally, we assume no external disturbances such as shocks or vibrations at the plant input and zero cross-coupling between the two axes.

\section{CONTROLLER DESIGN}

The control scheme is based on the architecture described in [6]. In order to exploit the inherent properties of the spiral reference, the components of the controller are tuned accordingly, while additionally, the controller is extended with frequency shifting peak filters. Two individual controllers with identical architecture are used, one for each axis of motion. The block diagram of the resulting control loop is depicted in Figure 3. The architecture comprises a linear quadratic regulator $K_{r}$ for cancelation of the second order resonance of the plant combined with a Kalman filter $K_{\text {est }}$ for state estimation. Due to the similarity and smoothness of the reference signal in both axes, both feed-forward components $K_{F F}$ are selected as the inverse of the respective dc gains, focusing on tracking
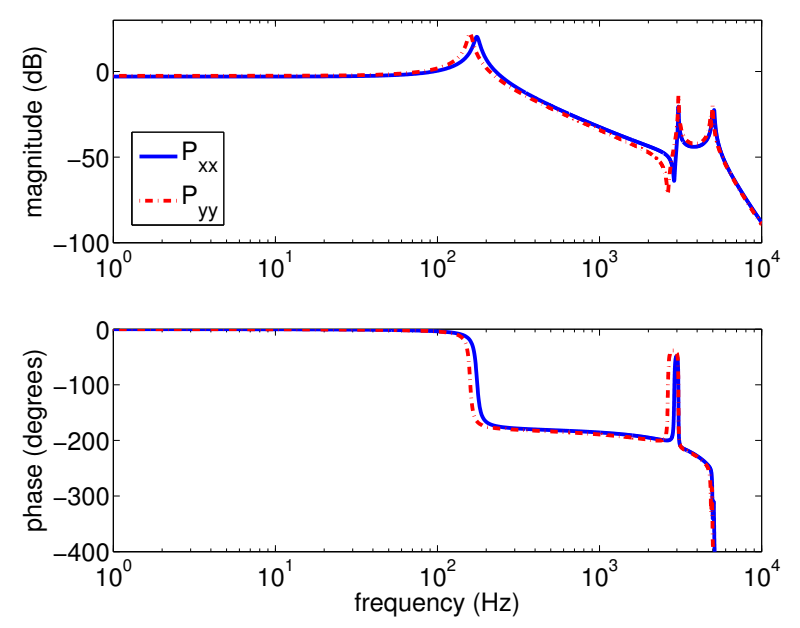

Fig. 2. MEMS-based microscanner model. 


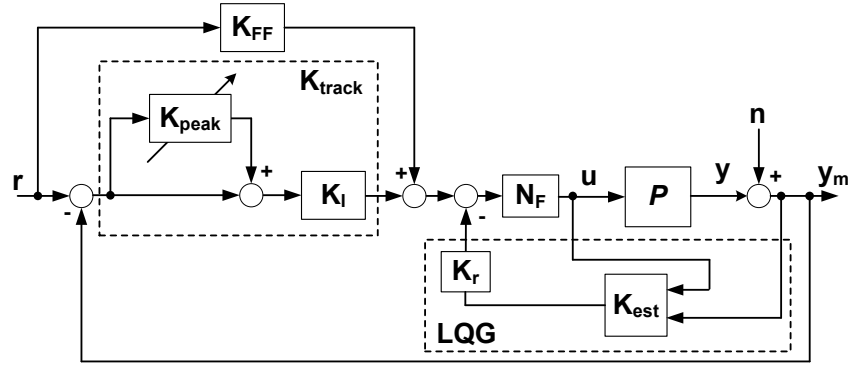

Fig. 3. Controller architecture block diagram.

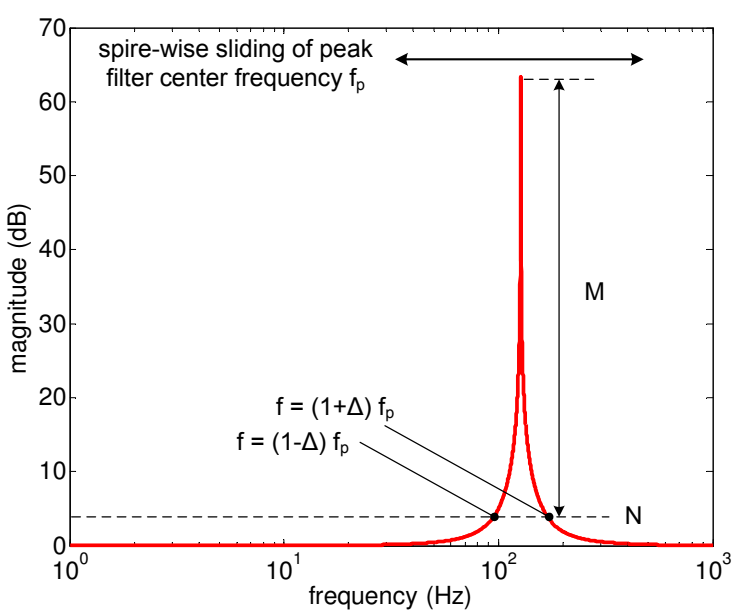

Fig. 4. Peak filter magnitude response.

efficiency. The tracking component consists of a pure integrator $K_{I}$ and a peak filter $K_{\text {peak }}$ with variable coefficients for enhanced tracking at the spire-determined frequency of interest. Finally, $N_{F}$ denotes the two notch filters per axis, used for compensation of the higher order resonances, while $n$ represents the combined measurement and quantization noise. The peak filter can be constructed according to [9]:

$$
K_{\text {peak }}(s)=\frac{s^{2}+2 \zeta_{1} \omega_{p} s+\omega_{p}^{2}}{s^{2}+2 \zeta_{2} \omega_{p} s+\omega_{p}^{2}}
$$

where $f_{p}=\omega_{p} /(2 \pi)$ the spire-wise center scan frequency which is updated according to Eq. 2 , and $\zeta_{1}, \zeta_{2}$ the damping ratios of the filter. The latter can be expressed in terms of the parameters $M, N, \Delta$, where $M$ denotes the magnitude at $f_{p}$, and $N$ the magnitude at the frequency points around the center frequency, which are determined by $\Delta$. The parameters are set individually, according to the desired magnitude response of the filter, and are illustrated qualitatively in Figure 4. For simulations, the discretized version of the filter is used, obtained by substituting $s=\frac{2}{T_{s c}} \frac{1-z^{-1}}{1+z^{-1}}$ in Eq. 3, where $T_{s c}$ the sampling period of the control loop.

The resulting transfer functions of the closed loop system are shown in Figure 5, where $T_{\text {ref }}$ relates the reference with the output, $S_{r e f}$ relates the error signal $y-r$ with the

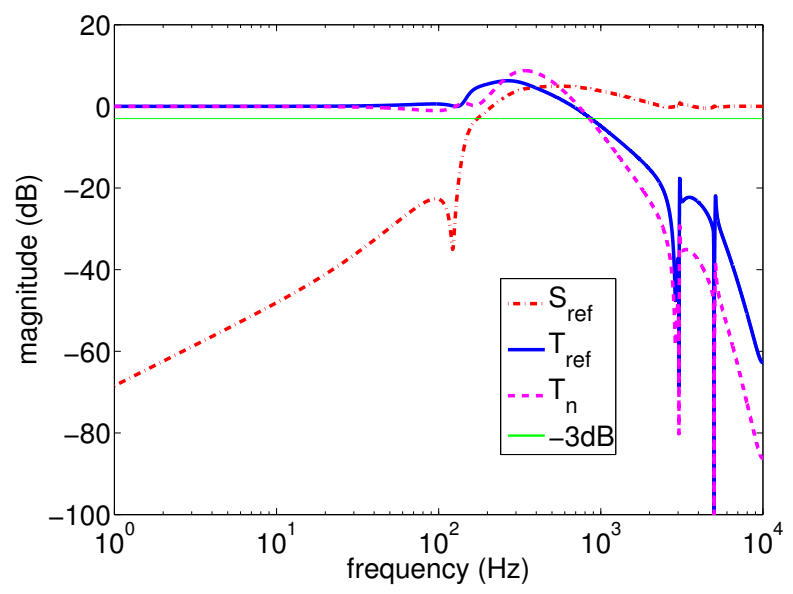

Fig. 5. Closed loop transfer functions for the $\mathrm{x}$-axis controller.

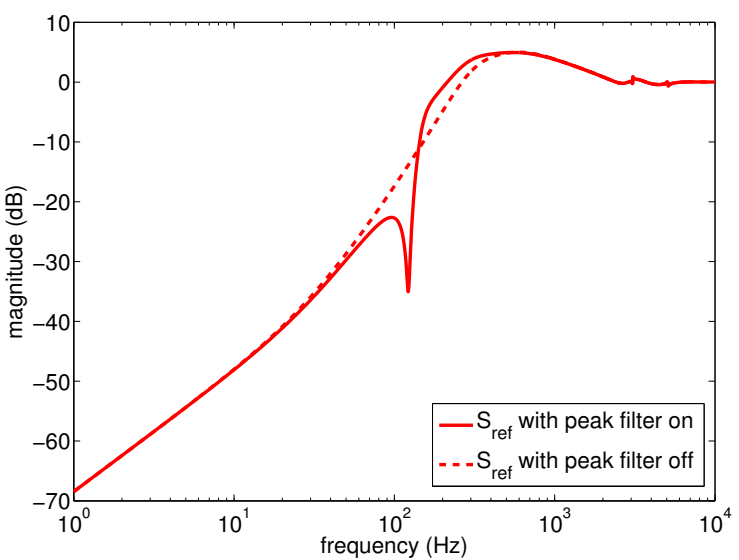

Fig. 6. Effect of peak filter in x-axis $S_{\text {ref }}$.

output and $T_{n}$ relates the noise signal with the output. The controller bandwidth is determined by the point where $S_{\text {ref }}$ crosses $-3 \mathrm{~dB}$ from below, which is approximately $190 \mathrm{~Hz}$ in this case. Notice also, that $T_{r e f}$ is not flat at the frequency region close to the end of the controller bandwidth. Nevertheless, $T_{\text {ref }}$ falls to $0 \mathrm{~dB}$ at the peak filter operation frequency yielding better tracking at that specific point. Figure 6 demonstrates the effect of the peak filter insertion, which causes an additional drop in $S_{r e f}$ at each spire-related frequency. Note, however, that the responses in the figures represent just time-snapshots of the transfer functions, since they are spire-dependent. The mathematical expressions of the transfer functions can be found in [6], where $K_{t r}$ should be substituted with $K_{\text {track }}=\left(1+K_{\text {peak }}\right) K_{I}$, in order to include the peak filter, and the gain of $K_{I}$ is reduced by half to offset the gain introduced by the peak filter structure. 

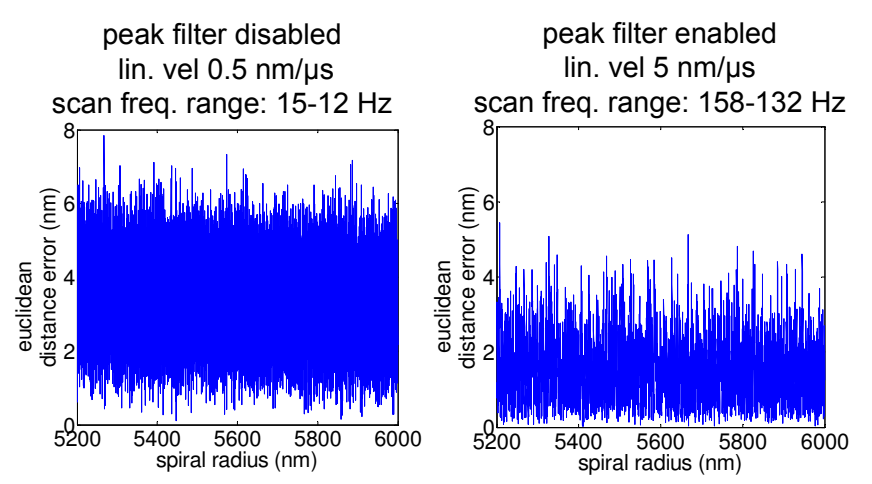

Fig. 7. Tracking error with respect to spiral radius.

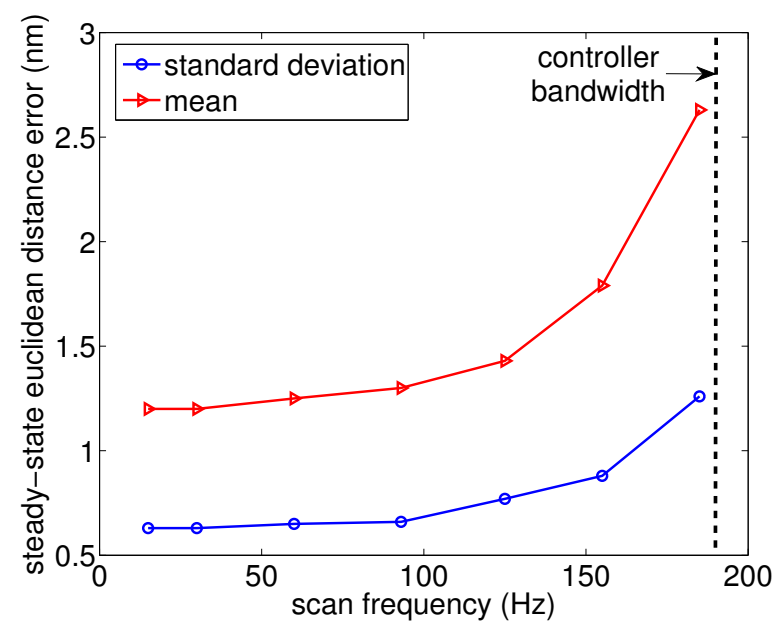

Fig. 8. Steady-state tracking error versus scan frequency with the peak filter enabled.

\section{PERFORMANCE RESULTS}

The tracking performance of the proposed architecture is evaluated using the steady-state $\mathrm{x} / \mathrm{y}$-plane euclidean distance between the reference position signal and the system output position as the error metric. The transient response of the system is not investigated in terms of the time required for the system to reach steady state, since this property depends highly on the approach/seek procedure used, which is outside the scope of this work. It is though expected, that the response becomes more oscillatory when the peak filter is included in the loop, which is due to the phase distortion introduced by the peak filter. Nevertheless, this delay is by far counterbalanced by the higher speed operation.

Figure 7 clarifies the superior tracking performance of the system when the peak filter is included in the loop. With the filter enabled, the tracking error falls below $5 \mathrm{~nm}$ even for a very high scan frequency range of $158-132 \mathrm{~Hz}$. This is clearly due to the additional gain provided by the peak filter structure at each specific scan frequency. When the peak filter is disabled, the tracking error meets the requirements for most nanoscale applications, only for low scan frequencies below $16 \mathrm{~Hz}$. For higher frequency scans, tracking error falls outside acceptable levels. Figure 8 presents aggregated performance results of the mean and standard deviation of the steady-state error versus increasing scan frequencies for 30 spires, starting from a spiral radius of $10 \mu \mathrm{m}$. Even at scan frequencies as high as $160 \mathrm{~Hz}$, the mean positioning error is well below 2 $\mathrm{nm}$ with a standard deviation of less than $1 \mathrm{~nm}$. Note that for this radius the scan frequencies correspond to constant linear velocities of $1,2,4,6,8,10$ and $12 \mathrm{~nm} / \mu \mathrm{s}$, which are well beyond previously reported velocities for the microscanner system or other closed loop systems with nanopositioners of similar bandwidth.

\section{CONCLUSIONS}

To sum up, a track-follow controller for achieving high-speed nanopositioning along archimedean spiral trajectories has been presented, analyzed and evaluated. It has been demonstrated that the controller achieves very high speed operation at scan frequencies near the controller bandwidth, while at the same time maintains the positioning accuracy in levels that meet the requirements of nanoscale applications.

\section{REFERENCES}

[1] S. Devasia, E. Eleftheriou, and S. O. R. Moheimani, "A survey of control issues in nanopositioning," IEEE Trans. on Control System Technology, vol. 15, no. 5, Sept. 2007.

[2] A. Pantazi et. al, "Probe-based ultrahigh-density storage technology," IBM J. Res. and Dev., vol. 52, no. 4/5, 2008.

[3] Bharat Bhushan, Handbook of Nanotechnology, Springer Verlag, Germany, January 2004.

[4] A. G. Kotsopoulos and T. A. Antonakopoulos, "Nanopositioning using the spiral of archimedes: The probe-based storage case," Mechatronics, vol. 20, no. 2, 2010.

[5] H. Ogawa Heitarso Nakajima, Compact Disc Technology, Ios Pr Inc, January 1993.

[6] A. Pantazi et. al, "Control of MEMS-based scanningprobe data storage devices," IEEE Transactions on Control System Technology, vol. 15, no. 5, Sept. 2007.

[7] A. Sebastian et. al, "Nanopositioning for probe-based data storage," IEEE Control Systems Magazine, 2008.

[8] M.A. Lantz et. al, "A vibration resistant nano-positioner for mobile parallel-probe storage applications," J. Microelectomech. Syst. 16(1), pp: 130 139, Feb. 2007.

[9] Abdulah Al Mamum, GuoXiao Guo, and Chao Bi, Hard Disk Drive Mechatronics and Control, CRC Press, 2007. 